\title{
Iterative Data Detection and Channel Estimation for Advanced TDMA Systems
}

\author{
Nikolai Nefedov, Member, IEEE, Markku Pukkila, Member, IEEE, Raphael Visoz, and \\ Antoine O. Berthet, Member, IEEE
}

\begin{abstract}
This letter presents a new receiver for $Q$-ary transmission, where all receiver blocks are embedded in an iterative structure. Packet data transmission in global systems for mobile communications (GSM) and enhanced data rates for global evolution (EDGE) are considered as examples. A low-complexity soft-insoft-out detector for EDGE is introduced and its modification suitable for iterative detection is derived. Application of iterative detection and channel estimation techniques in GSM/EDGE shows a significant performance enhancement. Additional improvement may be obtained if the iterative processing is applied to packet retransmission schemes.
\end{abstract}

Index Terms-Adaptive estimation, decision feedback equalizers, iterative detection, time-division multiple-access (TDMA) communications.

\section{INTRODUCTION}

$\mathbf{T}$ HIS LETTER presents a generic time-division multiple-access (TDMA) receiver for $Q$-ary signaling that performs iterative equalization [1] and estimation in a disjoint iterative fashion. Although this receiver design can be used for any TDMA system, it is particularly interesting for eight-phase-shift keying (PSK) modulation adopted in enhanced data rates for global evolution (EDGE). Recently, iterative (turbo) detection has gained a lot of interest with different approaches suggested. For example, the turbo detection (TD) with maximum a posteriori (MAP)-based detectors for EDGE is considered in [2]. However, the complexity of such detectors is prohibitively high for EDGE, entailing the need of suboptimal detectors. The delayed decision-feedback sequence estimator (DDFSE) with prefiltering is shown to give a reasonable tradeoff between performance and complexity [3], [4]. Since TD requires soft probabilistic information, the originally hard-output DDFSE has to be modified. In this letter, we first derive a low-complexity DDFSE-based detector with soft outputs suitable for iterative detection. To improve performance further, we introduce another iterative procedure closely connected to the turbo detector to perform channel estimation. Application of the TD for retransmission schemes brings additional gain.

Paper approved by C.-L. Wang, the Editor for Modulation Detection and Equalization of the IEEE Communications Society. Manuscript received April 9 , 2001. This paper was presented in part at the International Symposium on Turbo Codes, Brest, France, September 4-7, 2000, and in part at the IEEE Vehicular Technology Conference '01-Spring, Rhodes, Greece, May 2001.

N. Nefedov and M. Pukkila are with Nokia Research Center, Nokia Group, FIN-00045, Finland (e-mail: nikolai.nefedov@nokia.com; markku.pukkila@nokia.com).

R. Visoz is with France Telecom R\&D, 92130 Issy les Moulineaux, France (e-mail: raphael.visoz@ rd.francetelecom.com)

A. O. Berthet was with France Telecom R\&D, 92130 Issy les Moulineaux, France. He is now with Ecole Supérieure d'Electricité (SUPELEC), 91192 Gif-sur-Yvette, France (e-mail: Antoine.Berthet@ supelec.fr).

Digital Object Identifier 10.1109/TCOMM.2003.809218
The letter is organized as follows. Section II introduces notations and outlines the optimal and conventional receivers. A low-complexity soft-in-soft-out (SISO) detector suitable for TD and its extension to retransmission schemes are derived in Section III. A simple iterative channel estimation combined with the turbo detector is also proposed in the same section. Finally, simulation results for EDGE under different realistic scenarios are presented and discussed in Section IV.

\section{CONVEntional Receiver}

Let us consider a digital communication system where input data $\mathbf{u}=\left(u_{1}, \ldots, u_{K}\right)^{\top}$ are encoded by a channel encoder $\mathcal{C}$ resulting in a coded sequence $\mathbf{c}=\left(c_{1}, \ldots, c_{N}\right)^{\top}$. Coded bits are interleaved by an interleaver $\Pi$, punctured to match data rates to a transmitted format, and then grouped into $Q$-ary symbols $\left(Q=2^{q}\right)$ forming transmission bursts. Every burst a consists of $\tau$ symbols, including a known symbol training sequence $\mathbf{s}$ for channel estimation. A modulator $\Psi$ maps each burst into a complex-valued vector $\mathbf{z}=\left(z_{1}, \ldots, z_{\tau}\right)^{\top}$. At the output of the baseband-equivalent discrete-time channel $\mathbf{h}=\left(h_{0}, h_{1}, \ldots, h_{\mu}\right)^{\top}$, received samples are expressed as $y_{n}=\sum_{k=0}^{\mu} h_{k} z_{n-k}+w_{n}$, where $w_{n}$ is circularly complex Gaussian noise of variance $2 \sigma^{2}$ and considered independent identically distributed (i.i.d.) in the following.

According to the maximum-likelihood (ML) criterion applied to minimize block-error rate (BLER) for data block $\mathbf{u}$, the optimal receiver aims at identifying

$$
(\widehat{\mathbf{h}}, \widehat{\mathbf{u}})=\arg \max _{(\mathbf{h}, \mathbf{u})} \operatorname{Pr}(\mathbf{y} \mid \mathbf{h}, \mathbf{u}, \mathbf{s}, \Pi, \mathcal{C})
$$

given the received samples $\mathbf{y}=\left(y_{1}, \ldots, y_{\tau}\right)^{\top}$. Finding this optimal solution is prohibitively complex, consequently, a conventional receiver rather splits the problem into three separated ML tasks: 1) ML channel estimation: $\left.\widehat{\mathbf{h}}=\arg \max _{\mathbf{h}} \operatorname{Pr}(\mathbf{y} \mid \mathbf{h}, \mathbf{s}) ; 2\right)$ ML coded data detection: $\widehat{\mathbf{a}}=\arg \max \operatorname{Pr}(\mathbf{y} \mid \mathbf{a}, \widehat{\mathbf{h}})$; and 3) ML channel decoding: $\widehat{\mathbf{u}}=\arg \max _{\mathbf{u}} \operatorname{Pr}(\widehat{\mathbf{c}} \mid \mathbf{u}, \mathcal{C})$.

\section{ITERATIVE RECEIVER}

\section{A. Low-Complexity Turbo Detector}

The suboptimality due to separation of data detection and channel decoding can be partly recovered by the TD [1]. Similar to DDFSE [3], we describe here a decision-feedback SISO (DF-SISO) detector operating on a reduced complexity trellis, where only $\nu$ most recent symbols form the trellis state space $S$, and the earlier $\mu-\nu$ symbols are used through the embedded DF structure. Let $B$ denote the branch (or transition) space. Any trellis branch $b=\left(s^{\prime}, s, \mathbf{e}\right) \in B$ with $s^{\prime}, s \in S^{2}$, carries a label e which, at time $n$, represents an input symbol 


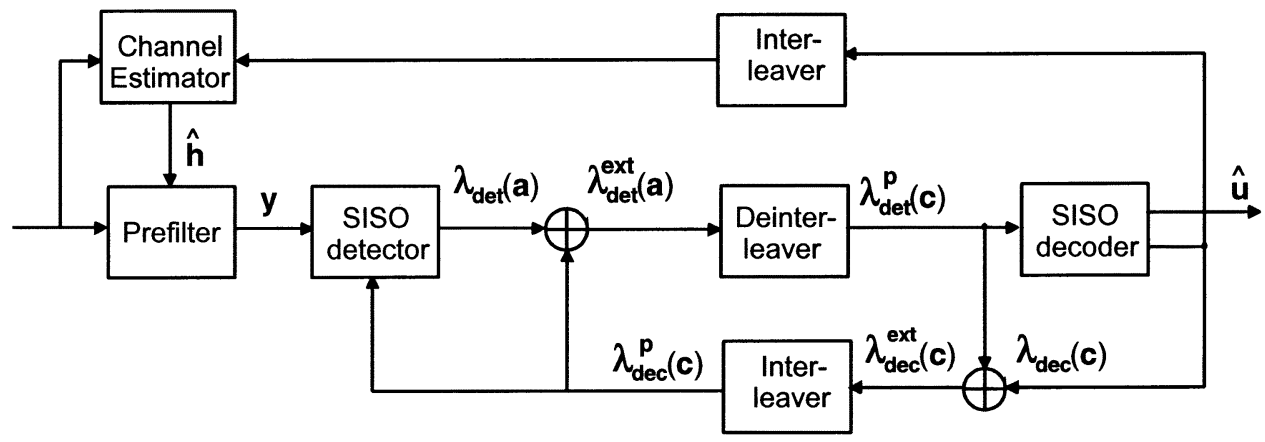

Fig. 1. Block diagram of iterative receiver.

$\mathbf{a}_{n}$ formed by bits $\left\{a_{n, 1}, a_{n, 2}, \ldots, a_{n, q}\right\}$. Each trellis path is a set of transitions starting from state $\varsigma$ at $n=0$ and terminating at state $\eta$ at $n=\tau$. An optimal bitwise detector would compute for $\forall n \in[1, \tau]$ and $\forall j \in[1, q]$ the $\log$ a posterior probability ratio (LAPR) on a symbol bit $a_{n, j}$, defined as $\lambda_{\operatorname{det}}\left(a_{n, j}\right)=\ln \left(\left(\operatorname{Pr}\left(a_{n, j}=1 \mid \mathbf{y}\right)\right) /\left(\operatorname{Pr}\left(a_{n, j}=0 \mid \mathbf{y}\right)\right)\right)$. The "max-log" approximation on soft decision outputs rather considers

$$
\lambda_{\operatorname{det}}\left(a_{n, j}\right) \cong \max _{\mathbf{a} \mid a_{n, j}=1}\{\ln p(\mathbf{a}, \mathbf{y})\}-\max _{\mathbf{a} \mid a_{n, j}=0}\{\ln p(\mathbf{a}, \mathbf{y})\}
$$

where $\{-\ln p(\mathbf{a}, \mathbf{y})\}$ is the trellis path metric associated with symbol sequence $\mathbf{a}$. To reduce the detector complexity, we can use only the forward recursion to form soft decisions. Introducing a decision delay $\delta$, terms (1) for $\varepsilon=0,1$ are then calculated as [5]

$$
-\ln \operatorname{Pr}\left(a_{n-\delta, j}=\varepsilon \mid \mathbf{y}\right) \cong \min _{b \in \mathrm{B} \mid \hat{a}_{n-\delta, j}^{\left(s^{\prime}, n-1\right)}=\varepsilon}\left\{\alpha_{n-1}\left(s^{\prime}\right)+\gamma_{n}(b)\right\}
$$

where $\gamma_{n}(b)$ is the DDFSE branch metric

$$
\gamma_{n}(b)=\frac{1}{2 \sigma^{2}}\left\|y_{n}-\sum_{k=0}^{\nu} \widehat{h}_{k} z_{n-k}-\sum_{k=\nu+1}^{\mu} \widehat{h}_{k} \widehat{z}_{n-k}^{\left(s^{\prime}, n-1\right)}\right\|^{2}
$$

$\alpha_{n-1}\left(s^{\prime}\right)$ is the accumulated metric of the best subpath starting from state $\varsigma$ and terminating in $s^{\prime}$ at $n-1$ and $\widehat{a}_{n-\delta, j}^{\left(s^{\prime}, n-1\right)}$ denotes the estimated symbol bit $a_{n-\delta, j}$ from the survivor path attached to $s^{\prime}$ at $n-1 . \alpha_{n}(s)$ can be recursively computed using the forward recursion similar to [6]

$$
\alpha_{n}(s)=\min _{b \in B^{(s)}}\left\{\alpha_{n-1}\left(s^{\prime}\right)+\gamma_{n}(b)\right\}
$$

where $B^{(s)}$ is a set of all branches $\left(s^{\prime}, s\right)$ terminating in state $s, B^{(s)} \subset B$; boundary conditions are $\alpha_{0}(\varsigma)=0, \alpha_{0}(s \neq$ $\varsigma)=\infty$ and $\alpha_{\tau}(\eta)=0, \alpha_{\tau}(s \neq \eta)=\infty$. Note that the soft-output processing retained here differs from a complete forward-backward strategy developed in [7]. The expression (2) involves the sequence $\left\{\mathbf{a}_{n-1}, \ldots, \mathbf{a}_{n-\nu}\right\}$ forming state $s^{\prime}$ and the sequence $\left\{\widehat{\mathbf{a}}_{n-\nu-1}^{s^{\prime}, n-1}, \ldots, \widehat{\mathbf{a}}_{n-\mu}^{s^{\prime}, n-1}\right\}$ obtained by reading off the survivor path terminating in $s^{\prime}$ at $n-1$. Since only the first $\nu$ channel taps form the trellis structure, it is beneficial for DF detectors to have the minimum phase channel impulse response (CIR) [3]. To meet this requirement, the usual practice is to put a prefilter before the detector that provides the minimum-phase CIR for the forward recursion.
A functional diagram of the reduced-complexity turbo detector is outlined in Fig. 1. The branch metric in (2) and (3) in the presence of independent prior probabilistic information on the transmitted symbol from the decoder is modified as $\gamma_{n}(b)-\gamma_{n}^{p}(b)$. Assuming perfect decorrelation between symbol bits after interleaving $\Pi$, we have

$$
\gamma_{n}^{p}(b)=\ln \operatorname{Pr}\left(a_{n, j}=e_{j}\right)+\sum_{l=1, l \neq j}^{q} \ln \operatorname{Pr}\left(a_{n, l}=e_{l}\right)
$$

where all $\operatorname{Pr}\left(a_{n, l}\right)$ are calculated from interleaved extrinsic probability ratios delivered by the decoder. At the output of the SISO detector, the bitwise soft output $\lambda_{\operatorname{det}}\left(a_{n-\delta, j}\right)$ is usually split into two parts

$$
\lambda_{\operatorname{det}}\left(a_{n-\delta, j}\right)=\lambda_{\operatorname{dec}}^{p}\left(a_{n-\delta, j}\right)+\lambda_{\operatorname{det}}^{\operatorname{ext}}\left(a_{n-\delta, j}\right)
$$

where $\lambda_{\mathrm{dec}}^{p}\left(a_{n-\delta, j}\right)=\ln \left(\left(\operatorname{Pr}\left(a_{n-\delta, j}=1\right)\right) /\left(\operatorname{Pr}\left(a_{n-\delta, j}=\right.\right.\right.$ $0)))$ is the log prior probability ratio on symbol bit $a_{n-\delta, j}$ provided by the SISO decoder; and $\lambda_{\operatorname{det}}^{\operatorname{ext}}\left(a_{n-\delta, j}\right)$ is the log extrinsic probability ratio (LEPR) that, in the case of the DF-SISO detector with forward-only recursion, may be expressed as

$$
\begin{aligned}
\lambda_{\operatorname{det}}^{\operatorname{ext}}\left(a_{n-\delta, j}\right)= & \min _{b \in B \mid \widehat{a}_{n-\delta, j-1}^{\left(s^{\prime}, n\right)=0}}\left\{\alpha_{n-1}\left(s^{\prime}\right)+\gamma_{n}^{\text {ext }}(b)\right\} \\
& -\min _{b \in B \mid \widehat{a}_{n-\delta, j}^{\left(s^{\prime}, n-1\right)}=1}\left\{\alpha_{n-1}\left(s^{\prime}\right)+\gamma_{n}^{\text {ext }}(b)\right\}
\end{aligned}
$$

with

$$
\gamma_{n}^{\mathrm{ext}}(b)=\gamma_{n}(b)-\sum_{l=1, l \neq j}^{q} \ln \operatorname{Pr}\left(a_{n, l}=e_{l}\right) .
$$

Note that the effect of prior probabilistic information in TD is twofold. First, it is accumulated during forward recursion as shown in (2)-(4). Second, it is explicitly present in (5). For binary modulation (e.g., Gaussian minimum-shift keying (GMSK), $q=1$ ), the second term in (5) is null. Hence, TD is expected to provide more gain for schemes with high-level modulation. Simulation results presented below confirm this conjecture. Additional improvement may be obtained if the TD is embedded into retransmission schemes, e.g., in the incremental redundancy (IR) scheme specified for EDGE [8]. Let $\lambda_{\operatorname{det}}^{\operatorname{ext}, k, n_{k}}$ be a sequence of extrinsic LEPRs on a coded block $\mathbf{c}^{(k)}$ at the output of the equalizer, $n_{k}$ is a number of turbo equalization/estimation iterations performed for the $k$ th retransmitted block. The proposed algorithm is the following. 


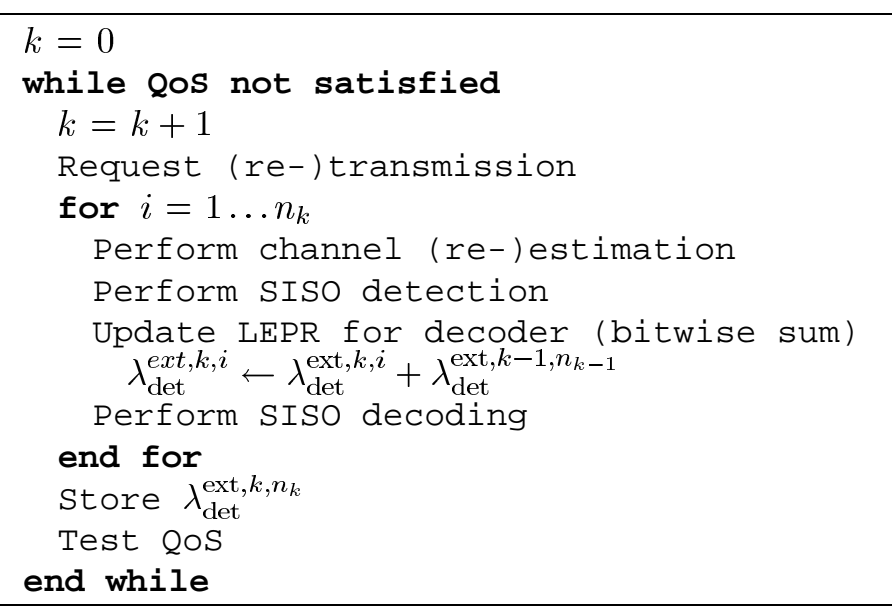

\section{B. Iterative Channel Estimation}

In many cases, the accuracy of the channel estimation, which is based on a relatively short training sequence $\mathbf{s}$, may be rather low. It may result in a significant performance degradation that will never be compensated by the turbo detector. This fact motivated us to use a decision-directed adaptive channel estimation during the iteration process, similar to [9], but with the difference of feeding back decoded symbols to the channel estimator (Fig. 1). Hence, the receiver relies on the hard decoded symbols $\widehat{\mathbf{c}}$ and the known training sequence $\mathbf{s}$ to form a new channel estimate. If all available data a are perfectly known at the receiver, then the ML channel estimate under an additive white Gaussian noise (AWGN) channel assumption is $\widehat{\mathbf{h}}^{\text {extend }}=\left(\mathbf{Z}^{\dagger} \mathbf{Z}\right)^{-1} \mathbf{Z}^{\dagger} \mathbf{y}$ where the Toeplitz matrix $\mathbf{Z}$ is formed by all data $\mathbf{a}$ [7], [10]. The channel re-estimation requires the matrix inverse for every transmitted block. It may be avoided by applying a simple least mean square (LMS) adaptive algorithm to update the estimate (see, for example, [11])

$$
\widehat{\mathbf{h}}^{(k+1)}=\widehat{\mathbf{h}}^{(k)}-\xi\left(\widehat{\mathbf{Z}}^{(k)}\right)^{\dagger}\left(\widehat{\mathbf{Z}}^{(k)} \widehat{\mathbf{h}}^{(k)}-\mathbf{y}\right)
$$

where $\widehat{\mathbf{h}}^{(k)}$ is a vector of channel coefficients estimated at the $k$ th iteration, $\widehat{\mathbf{Z}}^{(k)}$ is an estimated data matrix at the $k$ th iteration, and $\xi$ is a step size of the LMS algorithm.

\section{Simulation Results AND Discussion}

Performance of iterative receiver structures is evaluated for general packet radio system (GPRS) and enhanced GPRS (EGPRS) for different modulation-coding schemes (MCS). The receiver is made of a five-tap estimator (with the LMS adaptation rule (6) in the re-estimation round), the DF-SISO detector with $\nu=2$ and a max-log-MAP decoder. BLER performance is shown in Figs. 2 and 3 for the conventional receiver (solid lines), separately after iterative channel estimate update (dashed lines), and after the TD (lines with labels). Performance gains obtained from iterative data processing for typical urban (TU) channels and $3 \mathrm{~km} / \mathrm{h}$ mobile speed [8] are summarized in Tables I and II. As expected, the TD gain is getting lower at higher coding rates, but still remains noticeable (Table I). Even in case of MCS9, there is a 0.7-dB gain, mainly due to the presence of a coded packet header in each transmitted block. Table II summarizes improvement

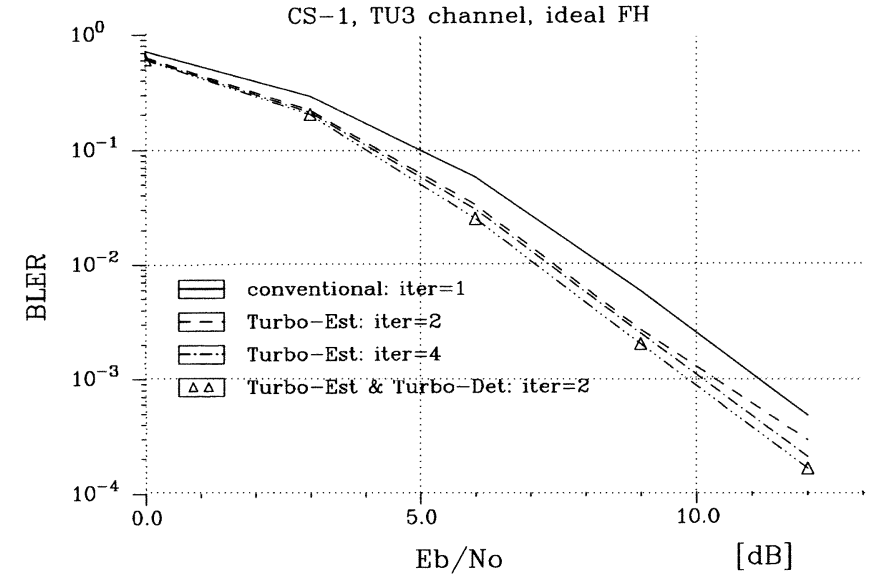

Fig. 2. Performance of GPRS iterative receiver (GMSK modulation) versus $E_{b} / N_{0}$ ( $E_{b}$ is energy per bit; $N_{0}$ is AWGN spectral density).

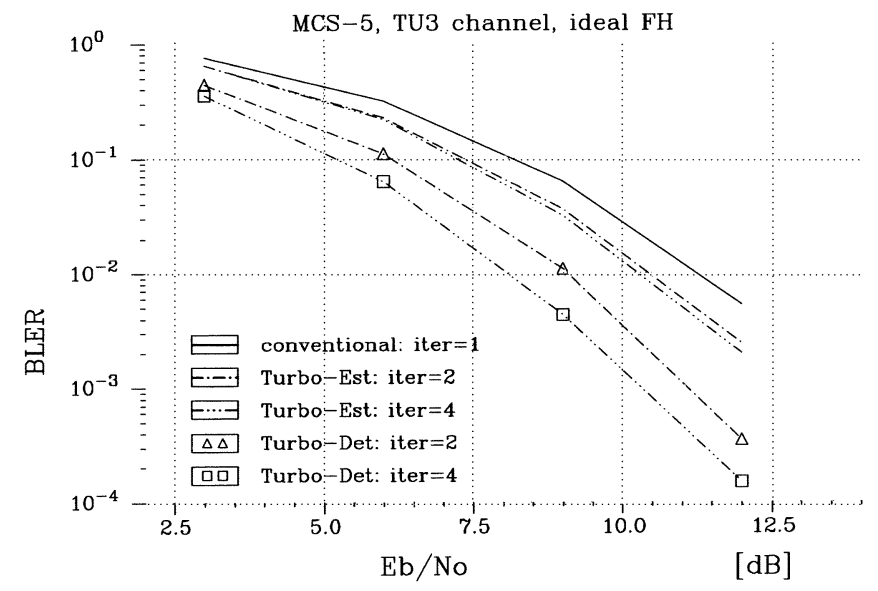

Fig. 3. Performance of EGPRS iterative receiver (8-PSK modulation) versus $E_{b} / N_{0}$.

TABLE I

EGPRS: TD GAIN IN DECIBELS FOR MCS5-MCS9 AT BLER $=10^{-1}$

\begin{tabular}{l|l|l|l|l|l}
\hline Coding scheme & MCS5 & MCS6 & MCS7 & MCS8 & MCS9 \\
\hline Code rate & 0.37 & 0.49 & 0.76 & 0.92 & 1.0 \\
\hline TD gain 2iter (4iter) & $2.2(3.2)$ & $2.1(3.1)$ & $1.9(2.4)$ & $0.9(1.7)$ & $0.7(1.4)$ \\
\hline
\end{tabular}

TABLE II

EGPRS: PERFORMANCE IMPROVEMENT IN DECIBELS DUE TO TD AND TE AT BLER $=10^{-2}$

\begin{tabular}{c|c|c|c|c}
\hline Coding scheme & Code rate & Modulation & TD 2it (4it) & TE 2it (4it) \\
\hline CS1 & 0.5 & GMSK & $0.2(0.3)$ & $1.0(1.1)$ \\
\hline MCS5 & 0.37 & 8PSK & $2.2(3.2)$ & $1.0(1.1)$ \\
\hline \hline Coding scheme & Code rate & Modulation & TD -TE one round each \\
\hline CS1 & 0.5 & GMSK & \multicolumn{2}{|c}{1.1} \\
\hline MCS5 & 0.37 & 8PSK & \multicolumn{2}{|c}{2.4} \\
\hline
\end{tabular}

obtained from turbo estimation (TE) and TD after two and four iterations, respectively. In the case of combined TD-TE, the results are shown for one round for each operation. In GPRS, the gain achieved by TE is around $1 \mathrm{~dB}$ at BLER $10^{-2}$, and the TD is able to provide only $0.2-\mathrm{dB}$ extra gain on top of that. The iterative estimation alone shows the same improvement in EGPRS. However, now the TD provides 2-3-dB gain, and that justifies the increase of decoder complexity to provide soft decisions. 


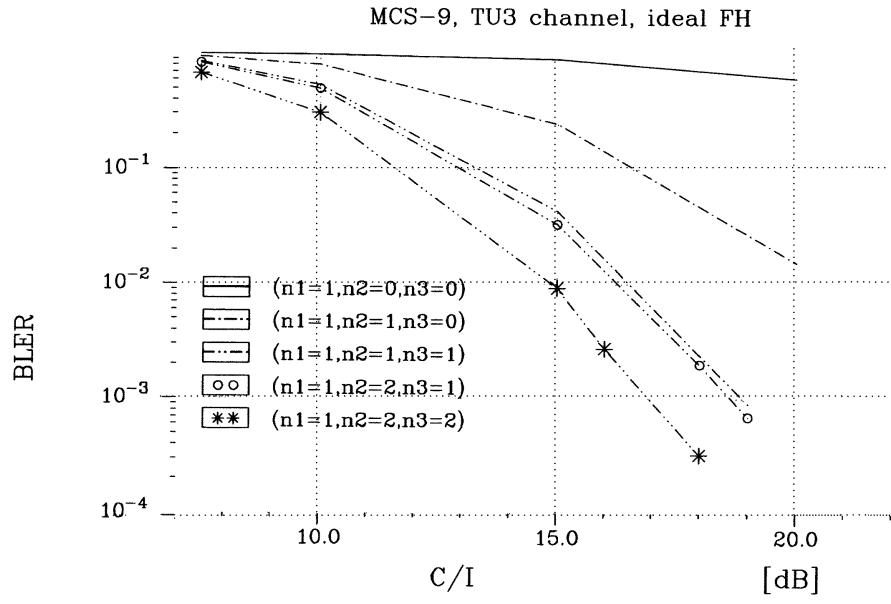

Fig. 4. Performance of TD combined with incremental redundancy versus carrier/interference ratio.

Simulation results for IR retransmission in EGPRS are shown in Fig. 4 for first, second, and third retransmissions with number of iterations $\left(n_{1}=1, n_{2}=0, n_{3}=0\right),\left(n_{1}=1, n_{2}=1, n_{3}=\right.$ $0),\left(n_{1}=1, n_{2}=1, n_{3}=1\right),\left(n_{1}=1, n_{2}=2, n_{3}=1\right)$, and finally $\left(n_{1}=1, n_{2}=2, n_{3}=2\right)$. As one can see, the proposed scheme provides $1.8 \mathrm{-dB}$ gain at BLER $10^{-2}$ if we compare retransmissions without $\left(n_{1}=1, n_{2}=1, n_{3}=1\right)$ and with $\left(n_{1}=1, n_{2}=2, n_{3}=2\right)$ TD-TE.

\section{CONCLUSION}

In this letter, a new approach for reception has been presented, where estimation, detection, channel decoding, and retransmission are performed in a disjoint iterative fashion. A low-complexity suboptimal detector and its modification suitable for TD are derived. It is shown that the TD brings more gain for high-order modulation schemes (EDGE), while less complex TE is more attractive for GSM. The suggested approach (TD-TE) provides up to 3.2-dB improvement in GSM/EDGE systems.

\section{REFERENCES}

[1] C. Douillard, M. Jézéchiel, C. Berrou, A. Picart, P. Didier, and A. Glavieux, "Iterative correction of intersymbol interference: Turbo equalization," Eur. Trans. Telecommun., vol. 6, pp. 507-511, Sept. 1995.

[2] V. Franz and G. Bauch, "Turbo detection for enhanced data GSM evolution," in Proc. IEEE VTC'99 Fall, vol. 4, Amsterdam, The Netherlands, Sept. 1999, pp. 2954-2958.

[3] A. Duel-Hallen and C. Heegard, "Delayed decision-feedback sequence estimation," IEEE Trans. Commun., vol. 37, pp. 428-436, May 1989.

[4] W. H. Gerstacker and R. Schober, "Equalization for EDGE mobile communications," Electron. Lett., vol. 36, pp. 189-191, Jan. 2000.

[5] J. Hagenauer and P. Hoher, "A Viterbi algorithm with soft-decision outputs and its applications," in Proc. IEEE Globecom'89, Nov. 1989, pp. $1680-1686$

[6] L. R. Bahl, J. Cocke, F. Jelinek, and J. Raviv, "Optimal decoding of linear codes for minimizing symbol error rate," IEEE Trans. Inform. Theory, vol. IT-20, pp. 284-287, Mar. 1974.

[7] A. O. Berthet, B. S. Unnal, and R. Visoz, "On iterative decoding of convolutionally encoded signals over multipath Rayleigh fading channels," IEEE J. Select. Areas Commun., vol. 19, pp. 1729-1743, Sept. 2001.

[8] ETSI digital cellular telecommunications system (phase 2+), Eur. Telecommun. Standards Inst., Sophia Antipolis, France, ser. GSM 05, rel. 2000.

[9] K.-H. Chang and C. N. Georghiades, "Iterative joint sequence and channel estimation for fast time-varying intersymbol interference channels," in Proc. IEEE ICC'95, Seattle, WA, June 1995, pp. 357-361.

[10] N. Nefedov and M. Pukkila, "Turbo equalization and iterative (turbo) estimation techniques for packet data transmission," in Proc. Int. Symp. Turbo Codes (ISTC'00), Brest, France, Sept. 2000, pp. 423-426.

[11] S. Haykin, Adaptive Filter Theory. Englewood Cliffs, NJ: PrenticeHall, 1996. 